\title{
Validation of manufacturers' laryngeal mask airway size selection standard: a large retrospective study
}

\author{
Yaoyao Ren ${ }^{1}$, Cuicui $\mathrm{Cao}^{2}$, Xuan Liang ${ }^{1}$, Zhihai Ju ${ }^{1}$, Ling Zhang ${ }^{3}$, Xu Cui ${ }^{1}$, Guyan Wang ${ }^{1}$ \\ ${ }^{1}$ Department of Anesthesiology, Beijing Tongren Hospital, Capital Medical University, Beijing, China; ${ }^{2}$ School of Management, Huazhong \\ University of Science and Technology, Wuhan, China; ${ }^{3}$ School of Public Health, Capital Medical University, Beijing, China \\ Contributions: (I) Conception and design: X Cui, G Wang, Y Ren; (II) Administrative support: X Cui, G Wang; (III) Provision of study materials or \\ patients: X Liang, Z Ju, Y Ren; (IV) Collection and assembly of data: C Cao, L Zhang; (V) Data analysis and interpretation: Y Ren; (VI) Manuscript \\ writing: All authors; (VII) Final approval of manuscript: All authors. \\ Correspondence to: Xu Cui; Guyan Wang. Department of Anesthesiology, Beijing Tongren Hospital, Capital Medical University, Beijing 100730, \\ China. Email: cuixubjtr@ccmu.edu.cn; guyanwang2006@163.com.
}

\begin{abstract}
Background: Laryngeal mask airway (LMA) is a prominent supraglottic airway device, widely used especially in difficult airway management. However, the LMA sizes recommended by the manufacturers are not always well matched in clinical practice, which leads to complications. To date, there are rare models to validate whether the manufacturers' standard is suitable for use in clinical practice.

Methods: A total of 58,956 patients undergoing general anesthesia using LMA device were included in the study between January 1, 2011 and December 31, 2018, to validate the adherence rate of LMA sizes according to the manufacturers' recommendations. A logistic regression analysis was performed based on the actual LMA size used in clinical practice to establish separately size selection guidelines with gender, weight, and age as variables in adults, adolescents, and children.
\end{abstract}

Results: LMA insertions were analyzed in 50,776 (86.1\%) adults, 3,548 (6\%) adolescents, and 4,632 $(7.9 \%)$ children. Suitability of manufacturers' recommendations was higher in children [male: $86.02 \%$; female: $85.09 \%$ ] than adults [male: $72.75 \%$; female: $78.13 \%$ ] or adolescents [male: $73.4 \%$; female: $70.79 \%$ ]. For adults and adolescents, LMA size was better predicted using the regression model rather than the manufacturers' recommendations [male adults: 82.4\% (81.16-83.57\%) vs. $73.21 \%(71.79-74.59 \%), \mathrm{P}<0.05$; female adults: $87.82 \%(86.65-88.9 \%)$ vs. $77.07 \%$ (75.6-78.48\%), $\mathrm{P}<0.05$; male adolescents: $79.45 \%$ (74.8683.4\%) vs. $72.05 \%$ (67.09-76.53\%), $\mathrm{P}<0.05$; female adolescents: $78.4 \%$ (71.11-84.31\%) vs. $72.22 \%$ (64.54$78.82 \%), \mathrm{P}<0.05]$. For children, there was equal performance suitability using the regression model and the manufacturers' recommendations.

Conclusions: The model-based guidelines may provide more accurate directions for LMA size selection for adolescents and adults than the manufacturers' weight-based recommendations, whereas the manufacturers' recommendation in children is consistent with clinical practice.

Keywords: Validation; model; manufacturer; laryngeal mask airway; size

Submitted Jun 20, 2020. Accepted for publication Nov 09, 2020.

doi: 10.21037/atm-20-4838

View this article at: http://dx.doi.org/10.21037/atm-20-4838

\section{Introduction}

There is increased use of supraglottic airway devices (SADs) in modern anesthesia and airway management. This type of device is popular because of its facility and effectiveness in difficult airway management and the advantages demonstrated, in contrast to the use of face mask and tracheal tube (1). Furthermore, the Society of Anesthesiologists has stressed the prominent role of SAD in difficult airway management $(2,3)$. As a crucial portion of SAD, the laryngeal mask airway (LMA), which was 
invented in the 1980s by Brain (4), consists of a triangular mask, an inflatable cuff, and a silicone connecting tube. It has gained wide acceptance in various clinical situations (5) as a substitute for the endotracheal tube (TT), with quicker and easier insertion, lower direct mechanical stimulation (6), and higher hemodynamic stability (7).

The LMA plays a vital role by forming an airtight seal enclosing the hypopharyngeal cavity (8). Thus, to ensure effective and safe ventilation, the proper LMA size is critically defined to have no or minimal leakage. Previous studies have demonstrated that an improper and too small size would result in leakage or aspiration (9), whereas a too large size may lead to sore throat (10) or injury of the lingual, recurrent laryngeal, and hypoglossal nerves (11).

Thus far, the most commonly used method for selecting the appropriate LMA size is still the manufacturers' recommendation based on weight (12). However, this method may not be the ideal choice. First, besides weight, other factors exist such as gender (13) and age (14) that may affect the oropharyngeal cavity and influence the LMA size selection. Second, the manufacturers' recommendation is based on cadaveric specimens, rather than clinical trials (15).

However, to date, there is still a paucity of data in the literature regarding the verification of manufacturers' LMA size selection recommendations. Therefore, this retrospective, large-sample study aims primarily to validate whether the manufacturers' recommendations are suitable for clinical practice. We present the following article in accordance with the TRIPOD reporting checklist (16) (available at http://dx.doi.org/10.21037/atm-20-4838).

\section{Methods}

The study was approved by the Beijing Tongren Hospital Institutional Review Board (No. TRECKY2016-020), and written informed consent was waived by the IRB due to the retrospective native of the paper. This study was conducted in accordance with the guidelines of the Declaration of Helsinki (as was revised in 2013). In addition, the privacy of all patients was protected. The ophthalmology and otorhinolaryngology departments of Beijing Tongren Hospital generally perform a considerable number of surgeries every year with patients coming from all regions of the country. LMA is accepted as the first choice of airway management in these types of surgeries, ensuring an enormous clinical database.

A retrospective review of patients' clinical information was obtained between January 1, 2011 and December 31,
2018 within the anesthesiology department, using the Anesthesia Information Management System (AIMS). The inclusion criteria were all of the patients who received general anesthesia with Flexible LMA as their airway device. This period was chosen because the AIMS was initially utilized in our hospital from January 1, 2011, and the data collection of 2019 is unfinished. By using the AIMS, patients' demographic data, for instance, age, gender, and the LMA size used, the failed first attempts at LMA insertion can all be retrieved. According to the guidelines of the anesthesiology department of Tongren Hospital, the attending physicians placed the LMAs on the patients, thereby ruling out the technical factor bias.

The patients were divided into three groups according to their age: the first group consisted of adults ( $>18$ years), the second group comprised adolescents evaluated as 10 to 18 years, and the third group included children (newborn to $<10$ years) (17). Each group was separated by gender into male and female. For assessing adherence to the manufacturers' suggestions, the weight limits underwent minor adjustments that aimed to eliminate overlap (Table 1). When the LMA size employed matched the manufacturers' suggestions, the proportion of patients in every weight group was defined as adherence (17).

\section{Statistical analysis}

A multinomial logistic regression was performed to identify the factors that might likely influence the LMA size selection as a function of weight, gender, and age. These models were built under the hypothesis that all of the patients included in this study accepted the most appropriate LMA size, just as it was recorded in the AIMS. A logistic regression was performed separately in each of the three age groups. The equation about model-based weight probability scores for LMA sizes for each group is listed in the Table S1. By using the cross-validation method (the holdout method), the data between January 1, 2011 and December 31, 2017 were collected as the development set, and the data between January 1, 2018 and December 31, 2018 were calculated as the validation set. The two different periods were determined using a larger database in building the model, thereby resulting in the achievement of better fitness and greater accuracy of the predicted parameters. Using the validation data set, the model's development data set parameters were verified. According to the predictors that achieved statistical significance in the development set, the LMA size model was constructed for the three different 
Table 1 Current weight-based laryngeal mask airway size recommendations by manufacturers and laryngeal mask airway size weight ranges used in the study

\begin{tabular}{lcc}
\hline Laryngeal mask airway size & Weight ranges prompted by manufactures & Manufactures' weight ranges applied in study \\
\hline 1 & $<5 \mathrm{~kg}$ & $<5 \mathrm{~kg}$ \\
1.5 & 5 to $10 \mathrm{~kg}$ & $\geq 5$ and $<10 \mathrm{~kg}$ \\
2 & 10 to $20 \mathrm{~kg}$ & $\geq 10$ and $<20 \mathrm{~kg}$ \\
2.5 & 20 to $30 \mathrm{~kg}$ & $\geq 20$ and $<30 \mathrm{~kg}$ \\
3 & 30 to $50 \mathrm{~kg}$ & $\geq 30$ and $<50 \mathrm{~kg}$ \\
4 & 50 to $70 \mathrm{~kg}$ & $\geq 50$ and $<70 \mathrm{~kg}$ \\
5 & $\geq 70 \mathrm{~kg}$ & $\geq 70 \mathrm{~kg}$ \\
\hline
\end{tabular}

Table 2 Demographic data of patients included in the study

\begin{tabular}{|c|c|c|c|c|c|c|c|}
\hline Age group & Total $n$ & Gender & $\mathrm{n}$ & Age (years) & $\mathrm{P}$ & Weight(kg) & $\mathrm{P}$ \\
\hline Adults & & Female & $23,215(45.7)$ & $49.0 \pm 0.1$ & & $61.1 \pm 0.1$ & \\
\hline \multirow[t]{2}{*}{ Adolescents } & 3,548 & Male & 2,489 (70.2) & $14.5 \pm 0.1$ & 0.4864 & $59.3 \pm 0.4$ & $<0.05$ \\
\hline & & Female & $1,059(29.8)$ & $14.6 \pm 0.1$ & & $52.4 \pm 0.4$ & \\
\hline Children & & Female & $1,700(36.7)$ & $5.3 \pm 0.1$ & & $20.9 \pm 0.2$ & \\
\hline
\end{tabular}

Data are presented as number (proportion) or mean \pm SD.

age groups. Concordance between the LMA size used in practice and the size forecasted by the manufacturers and model was calculated in the validation data set for each group.

A logistic regression analysis was performed using Stata 15.0 software (StataCorp LLC, USA). The descriptive statistics were analyzed to determine the clinical characteristics of the patients. Student's $t$-test was used to compare the continuous data. The chi-squared test was used to compare the proportions (SPSS 22.0 software, IBM, USA). A $\mathrm{P}<0.05$ was considered to be statistically significant.

\section{Results}

The data from January 1, 2011 to December 31, 2018 were collected using the AIMS in our hospital. During this period, we collected the data on all of the patients who used LMA as the first option of airway device. Finally, there were 58,956 LMA insertions for analysis, which consisted of $27,561(46.7 \%)$ male adults, $23,215(39.4 \%)$ female adults, 2,489 (4.2\%) male adolescents, 1,059 (1.8\%) female adolescents, $2932(5.0 \%)$ male children, and 1,700 (2.9\%) female children (Table 2).

The development data set included 50,492 cases, composed of 43,506 (86.1\%) adults, 3,021 (6\%) adolescents, and 3,965 (7.9\%) children. These cases were further divided by gender into six groups: male adults, female adults, male adolescents, female adolescents, male children, and female children. The adherence rate to the manufacturers' LMA size recommendations was calculated for these six groups (Table 3). The suitability of manufacturers' recommendations was higher in children [male: 2,159/2,510 (86.02\%); female: $1,238 / 1,455(85.09 \%)$ ] than adults [male: $17,198 / 23,641$ (72.75\%); female: 15,520/19,865 (78.13\%)] or adolescents [male: 1,559/2,124 (73.4\%); female: 635/897 (70.79\%); $\mathrm{P}<0.05$ for children $v$ s. adults or adolescents]. Furthermore, there were several low values: the adherence rate for male adults using LMA 3 was $11.83 \%$, the adherence rate for female adults using LMA 3 was $25.56 \%$, the adherence rate for female adults using LMA 5 was $37.32 \%$, the adherence rate for male adults using LMA 6 
Table 3 Adherence rate to manufacturers' laryngeal mask airway size recommendations

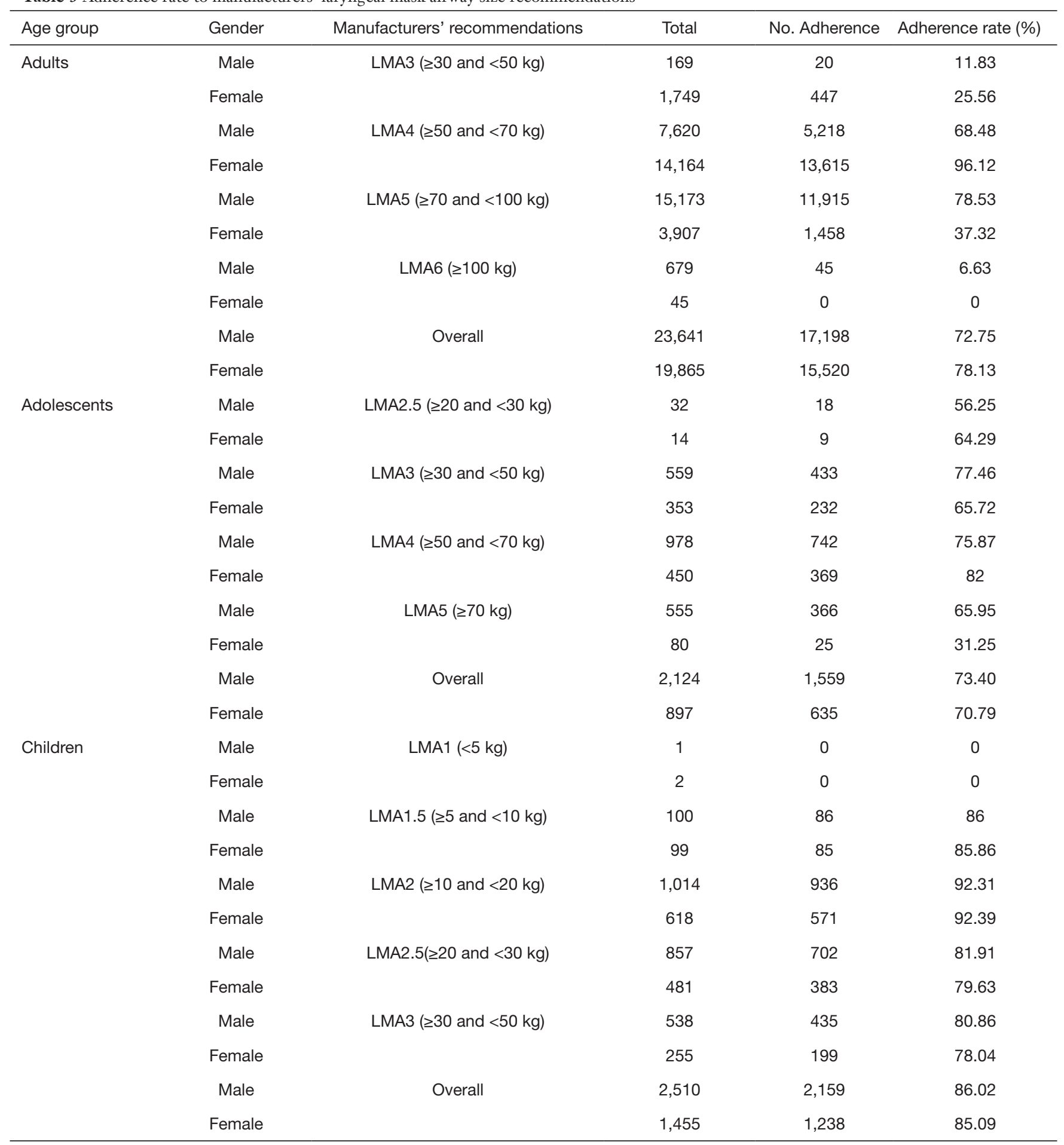

was $6.63 \%$, the adherence rate for female adolescents using LMA 5 was $31.25 \%$.

Therefore, a logistic regression analysis was performed based on the actual LMA size used in clinical practice aiming to build more accurate LMA size selection guidelines. Figure 1 shows the probability scores for LMA sizes by patient weight for each group. The model-based weight range recommendations in the LMA sizes are listed in Table 4. 

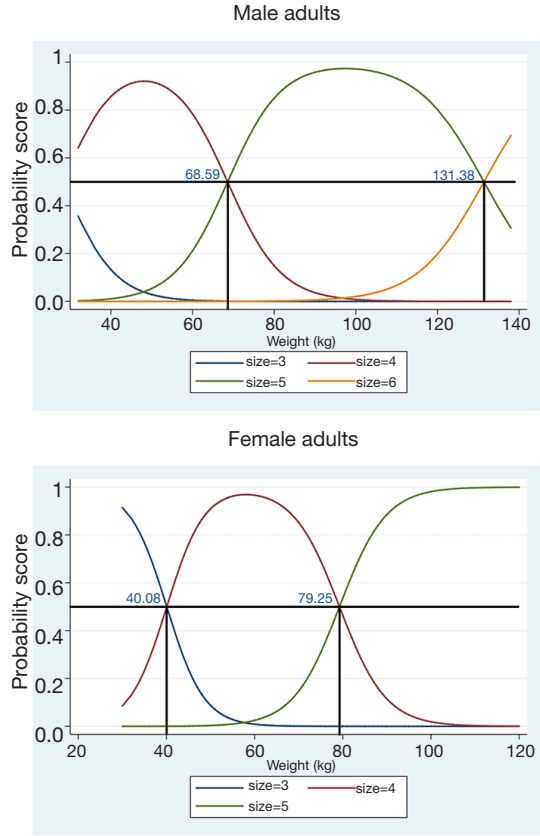

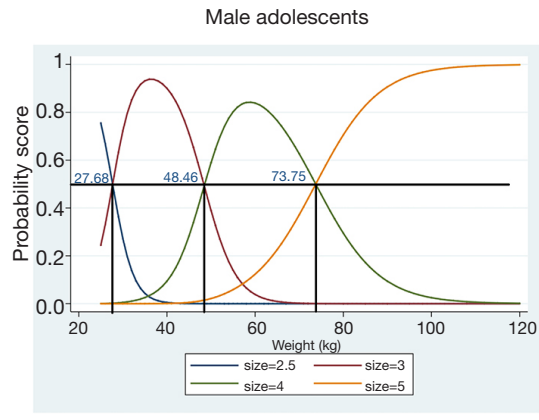

Female adolescents

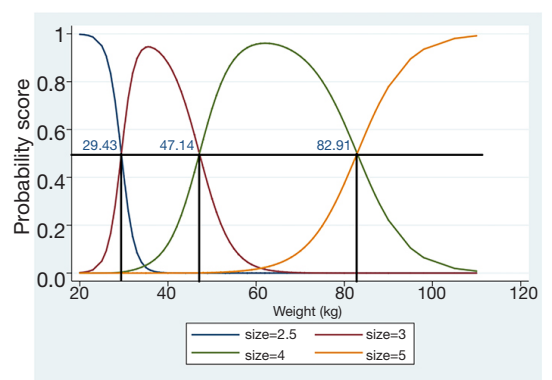

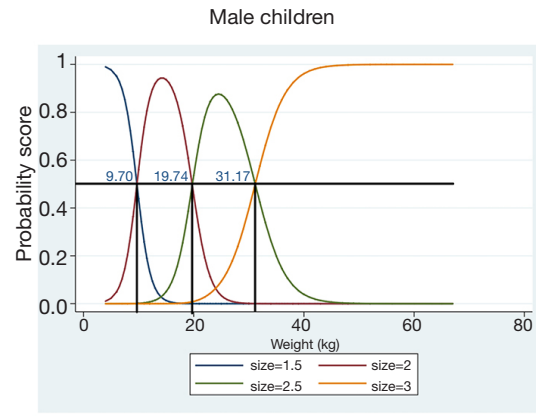

Female children

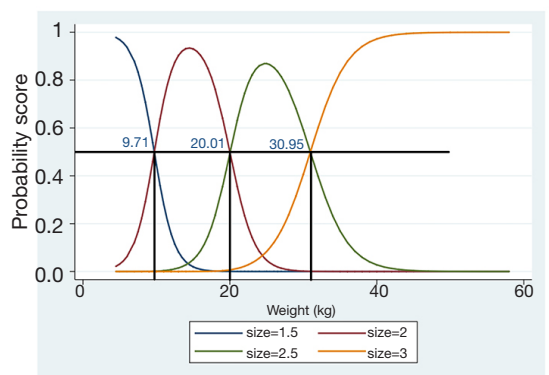

Figure 1 Model-based weight ranges for laryngeal mask airway sizes. Model-based weight probability scores (weight ranges) for LMA sizes for three age groups and gender. The vertical lines in the graphs are the model-based borders of the weight ranges (at the interception of the $50 \%$ probability score of the LMA sizes).

Table 4 The model-based weight range recommendations in the laryngeal mask airway (LMA) sizes

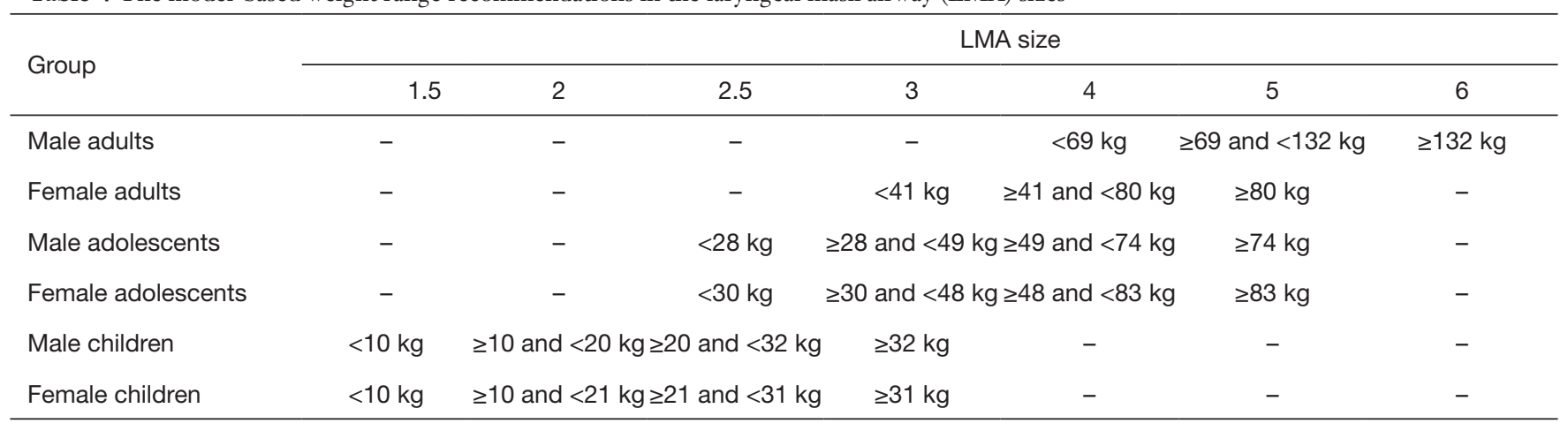

For adolescents and adults, the weight ranges of the model differed from the manufacturers' recommendations while the weight ranges for children were well matched to the manufacturers' recommendations. The adherence rate of LMA whose size selected according to the result of the regression model was calculated (Table 5) and compared with the manufacturers' recommendation. For adults and adolescents, the LMA size was better predicted using the regression model than the manufacturers' recommendations [male adults: $82.4 \%(81.16-83.57 \%)$ vs. $73.21 \%$ (71.79-
74.59), $\mathrm{P}<0.05$; female adults: $87.82 \%(86.65-88.9 \%)$ vs. 77.07 (75.6-78.48\%), $\mathrm{P}<0.05$; male adolescents: $79.45 \%$ (74.86-83.4\%) vs. 72.05 (67.09-76.53\%), $\mathrm{P}<0.05$; female adolescents: $78.4 \%(71.11-84.31 \%)$ vs. $72.22 \%(64.54-$ $78.82 \%), \mathrm{P}<0.05]$. For children, the regression model and the manufacturers' recommendations performed equally.

The validation set included 8,464 cases, and the distributions of gender, age, and weight were similar to the development set. Table 6 summarizes the concordance between the development and the validation sets for the 
Table 5 Adherence rate of the prediction by regression model

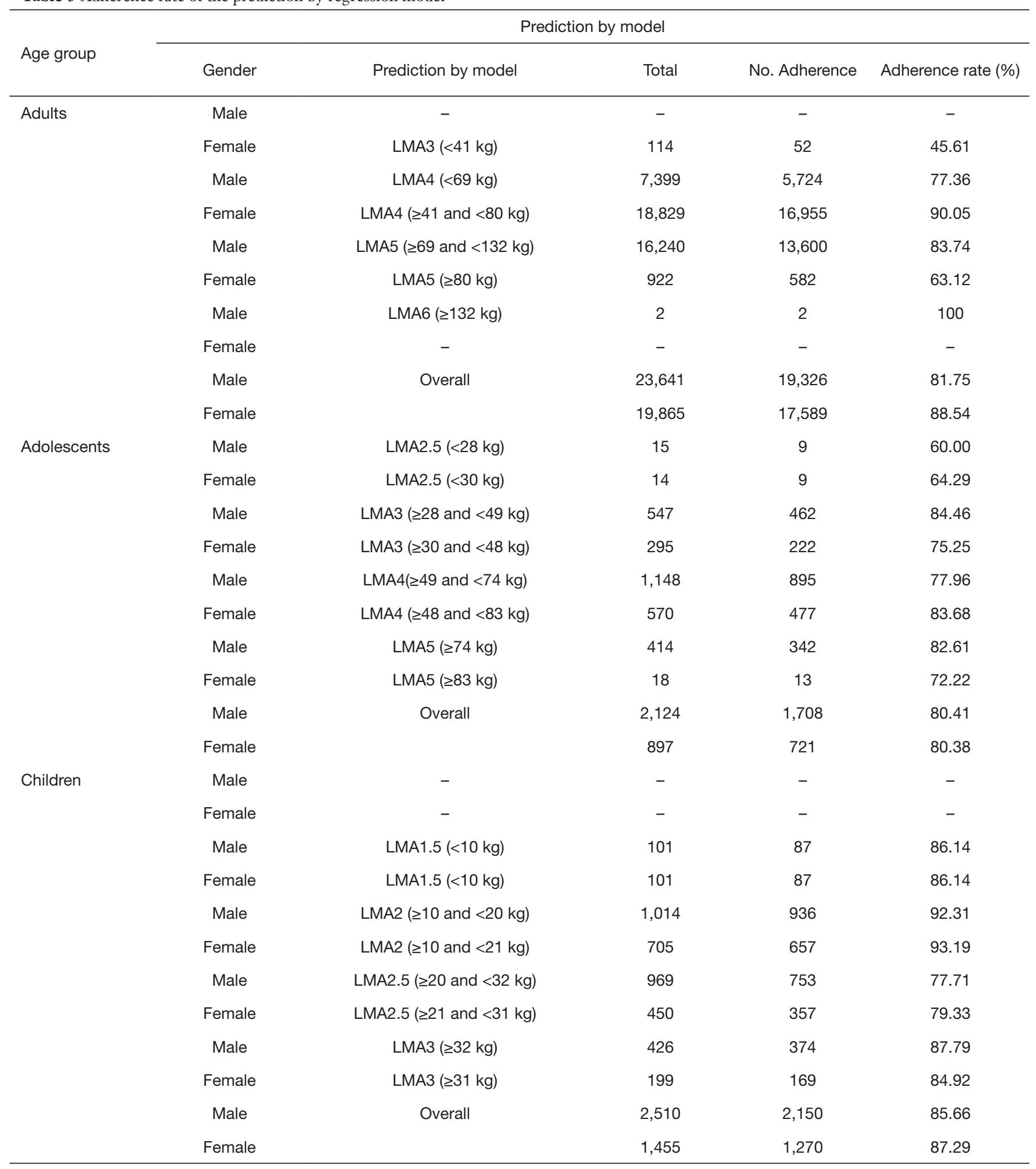


Table 6 Concordance (shown as percentage with 95\% confidence interval) between laryngeal mask airway sizes predicted by the manufacturers' recommendation and the regression model for each age group

\begin{tabular}{lcccccc}
\hline & \multicolumn{2}{c}{ Manufacturers' recommendation } & & \multicolumn{2}{c}{ Prediction by model } \\
\cline { 2 - 3 } \cline { 6 - 7 } & \multicolumn{2}{c}{ Development set } & Validation set & & Development set & Validation set \\
\hline Male adults & $72.8 \%(72.2 \%$ to $73.3 \%)$ & $73.2 \%(71.8 \%$ to $74.6 \%)$ & & $81.8 \%(81.3 \%$ to $82.2 \%)$ & $82.4 \%(81.2 \%$ to $83.6 \%)$ \\
Female adults & $78.1 \%(77.6 \%$ to $78.7 \%)$ & $77.1 \%(75.6 \%$ to $78.5 \%)$ & & $88.5 \%(88.1 \%$ to $89.0 \%)$ & $87.8 \%(86.7 \%$ to $89.0 \%)$ \\
Male adolescents & $73.4 \%(71.5 \%$ to $75.3 \%)$ & $72.1 \%(67.1 \%$ to $76.5 \%)$ & & $80.4 \%(78.6 \%$ to $82.1 \%)$ & $79.5 \%(74.9 \%$ to $83.4 \%)$ \\
Female adolescents & $70.8 \%(67.7 \%$ to $73.7 \%)$ & $72.2 \%(64.5 \%$ to $78.8 \%)$ & & $80.4 \%(77.6 \%$ to $82.9 \%)$ & $78.4 \%(71.1 \%$ to $84.3 \%)$ \\
Male children & $86 \%(84.6 \%$ to $87.3 \%)$ & $85.6 \%(81.8 \%$ to $88.7 \%)$ & & $85.7 \%(84.2 \%$ to $87.0 \%)$ & $84.4 \%(80.5 \%$ to $87.6 \%)$ \\
Female children & $85.1 \%(83.1 \%$ to $86.9 \%)$ & $86.9 \%(81.9 \%$ to $90.8 \%)$ & & $87.3 \%(85.4 \%$ to $88.9 \%)$ & $89.0 \%(84.2 \%$ to $92.5 \%)$ \\
\hline
\end{tabular}

manufacturer-based and model-based recommendations. The prediction of the appropriate LMA size selection exhibited greater accuracy in the model than according to the manufacturers' suggestions for adults and adolescents $(\mathrm{P}<0.05)$, except for children.

\section{Discussion}

The results of this large retrospective study demonstrated that for adults and adolescents, both gender and weight were significant factors for predicting the LMA sizes, which was likely due to the gender differences in upper airway structure between men and women. First, the pharyngeal airway is longer in healthy men compared to women independent of height (18-20). Second, the volume of soft tissue was larger in men compared to women (21). It was reported that the soft tissue surrounding the dentofacial skeleton had a prominent influence on the pharyngeal space (22). Third, the neck circumference was different between men and women, which influences the variations in the caliber of the upper airway (23). However, only weight played a significant role in predicting the LMA size for children. A probable reason is that the development of secondary sexual characteristics in children $<10$ years of age had not yet begun. Another likely reason is that the number of sizes available in children is greater with each size corresponding to a smaller weight range. In conclusion, the model-based weight ranges have a more accurate prediction of LMA size selection by using two variables: gender and age, compared with the manufacturers' guidelines, which include weight solely as a variable.

Compared to TT, the LMA as a routine airway management device has gained more and more popularity and acceptance in clinical practice with a series of advantages.
First, the LMA plays its role with less adverse perioperative respiratory events, such as laryngospasm, laryngeal edema, bronchospasm, bucking, coughing, soft tissue trauma, sore throat, and so on (24). Second, the LMA provides a more stable intraocular pressure that benefits patients undergoing ophthalmic surgery (25) and offers less hemodynamic instability during induction of and emergence from anesthesia (26) in patients with heart disease. Third, the low failure rate of Classic LMA ranges from $0.19 \%$ to $4.7 \%$ without the required visualization of the larynx $(27,28)$.

To the best of our knowledge, there are rare, large, retrospective studies in the literature, validating the manufacturers' LMA size selection standard for use in clinical practice, although various studies have been performed. Voyagis et al. and Berry et al. found that gender, height, and age all had an influence on the LMA size selection by affecting the oropharyngeal cavity $(9,13)$. Tang et al. (29) performed a randomized, single-blinded study to research the optimal size for Ambu ${ }^{\circledR}$ LMA (ALMA), and they recommended size 4 ALMA as the appropriate size for Malaysian adults. These findings were different from the manufacturer's suggestions. Unexpectedly, we found that the initial manufacturer's guidelines were based on cadaveric specimens of Caucasian adults $(6,15)$ rather than vast clinical trials, and that the correlation between hypopharynx and weight was inconsistent $(30,31)$.

The inappropriate recommendation caused a number of LMA failures and relevant complications in clinical practice, for instance, leakage, glottis impaction, and sore throat (32). As a result of the LMA leakage, shown as high airway pressure and inadequate ventilation, the patient may need to suspend and exchange a different size LMA or even TT to ensure that he or she receives sufficient ventilation. For this reason, there exist several other 
potential risks for patients. First, the surgery field may be polluted if the operative site is around the face. Second, the medical cost will be unnecessarily exorbitant Therefore, it is critical to validate the manufacturers' guidelines using a large retrospective study because all of those unwanted morbidities might be prevented if we could select the optimal size LMA. To the best of our knowledge, Avidan et al. conducted a retrospective analysis of 20,893 cases to validate the manufacturers' weight-based recommendations and found that the weight ranges used by the manufacturers were suitable for children, and unsuitable for adults and adolescents (17). They proposed a different weight range combined with gender and weight. However, their research results were different from ours, mainly reflected in the recommended weight ranges of LMA sizes. There are two possible reasons. First, the ethnicities of the study population are different. The study by Gu et al. noted significant differences in craniofacial morphology among individuals of Asian and Caucasian ancestries. Besides, there existed considerable gender differences in both ethnic samples. The main difference between Asian and Caucasian populations for young adults involved smaller facial and linear dimensions in the Asian population and a more hyperdivergent facial pattern in Asian females and males. As for soft tissue data, the Asian population has more protrusive upper and lower lips compared with Caucasians (33). Furthermore, Alves et al. (34) and Wang et al. (35) found that there was a correlation between the vertical skeletal angle and the airway width using 3D measurements. Notably, because of the different oropharyngeal anatomic structures of Asians and Caucasians, the use of the supraglottic airway would be different. A previous study showed that the use of a larger-sized PLMA (ProSeal ${ }^{\mathrm{TM}}$ Laryngeal Mask Airway) would provide a better glottic seal for Asians compared with Caucasians, likely due to the cephalometric differences between the two distinct categories (36). Distorted anatomy and small mandibular spaces could be used to predict difficulty in placing the supraglottic devices (37). Second, the sample size of the two studies is different. Therefore, it is questionable whether the manufacturers' guidelines derived from cadaveric specimens of Caucasians and the weight ranges calculated by Avidan et al. (17) are appropriate for all clinical practice.

In recent years, several novel methods have been proposed for predicting the size of LMA. The study by Zhu et al. revealed that the size selection of LMA according to cricoids-mental distance achieved a better airway seal and a higher success rate during the initial insertion compared with the traditional weight-based method (38). Haliloglu et al. found that the size selection of LMA based on the patient's auricle size was a useful substitute for the manufacturers' guidelines (39).

This study has several limitations. First, an obvious drawback in this study is its retrospective design, which is considered inferior to a prospective research. The retrospective design of this study rendered it impossible to analyze items of interest and made it possible to assess only standardized items included in daily clinical practice. Second, the LMA calculated was not distinguished by species, whereas there were small structural differences among the Classic LMA, LMA ProSeal, Flexible LMA, and LMA Supreme. The LMA used in this study was Flexible LMA, which was the main type of LMA used in our hospital. Third, as mentioned earlier, the height (9) and increased body mass index (BMI) (40), which is calculated by both weight and height, also have an impact on the decision of the LMA size; however, the heights of many patients were not recorded. Further evaluation should be performed to determine the correlation between LMA size and height or BMI.

\section{Conclusions}

The model-based guidelines according to our multinomial logistic regression analysis have a more accurate prediction of LMA size selection in adults and adolescents simultaneously using age, gender, and weight as the chosen variables, compared with the manufacturers' weight-based categories. Meanwhile, the manufacturers' recommendations for LMA size in children are consistent with our model-based guidelines and clinical practice. This study may be helpful for reducing complications caused by inappropriate LMA size and for establishing more accurate recommendations for LMA size selection.

\section{Acknowledgments}

Funding: This study is funded by Beijing Hospitals Authority Clinical Medicine Development of Special Funding Support, code: ZYLX202103.

\section{Footnote}

Reporting Checklist: The authors have completed the TRIPOD reporting checklist. Available at http://dx.doi. org/10.21037/atm-20-4838 
Data Sharing Statement: Available at http://dx.doi. org/10.21037/atm-20-4838

Peer Review File: Available at http://dx.doi.org/10.21037/ atm-20-4838

Conflicts of Interest: All authors have completed the ICMJE uniform disclosure form (Available at http://dx.doi. org/10.21037/atm-20-4838). The authors have no conflicts of interest to declare.

Ethical Statement: The authors are accountable for all aspects of the work in ensuring that questions related to the accuracy or integrity of any part of the work are appropriately investigated and resolved. This study was conducted in accordance with the guidelines of the Declaration of Helsinki (as was revised in 2013). This study was approved by the institutional ethics board of the Ethics Committee of Beijing Tongren Hospital (No. TRECKY2016-020), and written informed consent was waived due to the retrospective native of the paper.

Open Access Statement: This is an Open Access article distributed in accordance with the Creative Commons Attribution-NonCommercial-NoDerivs 4.0 International License (CC BY-NC-ND 4.0), which permits the noncommercial replication and distribution of the article with the strict proviso that no changes or edits are made and the original work is properly cited (including links to both the formal publication through the relevant DOI and the license). See: https://creativecommons.org/licenses/by-nc-nd/4.0/.

\section{References}

1. McIntyre JW. Oropharyngeal and nasopharyngeal airways: I (1880-1995). Can J Anaesth 1996;43:629-35.

2. Frerk C, Mitchell VS, McNarry AF, et al. Difficult Airway Society 2015 guidelines for management of unanticipated difficult intubation in adults. Br J Anaesth 2015;115:827-48.

3. Apfelbaum JL, Hagberg CA, Caplan RA, et al. Practice guidelines for management of the difficult airway: an updated report by the American Society of Anesthesiologists Task Force on Management of the Difficult Airway. Anesthesiology 2013;118:251-70.

4. Brain AI. The laryngeal mask--a new concept in airway management. Br J Anaesth 1983;55:801-5.

5. Jolliffe L, Jackson I. Airway management in the outpatient setting: new devices and techniques. Curr Opin Anaesthesiol 2008;21:719-22.

6. van Zundert TC, Brimacombe JR, Ferson DZ, et al. Archie Brain: celebrating 30 years of development in laryngeal mask airways. Anaesthesia 2012;67:1375-85.

7. Saraswat N, Kumar A, Mishra A, et al. The comparison of Proseal laryngeal mask airway and endotracheal tube in patients undergoing laparoscopic surgeries under general anaesthesia. Indian J Anaesth 2011;55:129-34.

8. Asai T, Morris S. The laryngeal mask airway: its features, effects and role. Can J Anaesth 1994;41:930-60.

9. Berry AM, Brimacombe JR, McManus KF, et al. An evaluation of the factors influencing selection of the optimal size of laryngeal mask airway in normal adults. Anaesthesia 1998;53:565-70.

10. Brain AI, Howard D. Lingual nerve injury associated with laryngeal mask use. Anaesthesia 1998;53:713-4.

11. Ahmad NS, Yentis SM. Laryngeal mask airway and lingual nerve injury. Anaesthesia 1996;51:707-8.

12. Zahoor A, Ahmad N, Sereche G, et al. A novel method for laryngeal mask airway size selection in paediatric patients. Eur J Anaesthesiol 2012;29:386-90.

13. Voyagis GS, Batzioulis PG, Secha-Doussaitou PN. Selection of the proper size of laryngeal mask airway in adults. Anesth Analg 1996;83:663-4.

14. Rommel N, Bellon E, Hermans R, et al. Development of the orohypopharyngeal cavity in normal infants and young children. Cleft Palate Craniofac J 2003;40:606-11.

15. Harnett M, Kinirons B, Heffernan A, et al. Airway complications in infants: comparison of laryngeal mask airway and the facemask-oral airway. Can J Anaesth 2000;47:315-8.

16. Heus P, Reitsma JB, Collins GS, et al. Transparent Reporting of Multivariable Prediction Models in Journal and Conference Abstracts: TRIPOD for Abstracts. Ann Intern Med 2020. [Epub ahead of print].

17. Avidan A, Eden A, Reider E, et al. Multicentre validation of manufacturers' weight-based recommendations for laryngeal mask airway size choice in anaesthetic practice: A retrospective analysis of 20,893 cases. Eur J Anaesthesiol 2015;32:432-8.

18. Malhotra A, Huang Y, Fogel RB, et al. The male predisposition to pharyngeal collapse: importance of airway length. Am J Respir Crit Care Med 2002;166:1388-95.

19. Segal Y, Malhotra A, Pillar G. Upper airway length may be associated with the severity of obstructive sleep apnea syndrome. Sleep Breath 2008;12:311-6.

20. Shigeta Y, Ogawa T, Tomoko I, et al. Soft palate length 
and upper airway relationship in OSA and non-OSA subjects. Tex Dent J 2013;130:203-11.

21. Whittle AT, Marshall I, Mortimore IL, et al. Neck soft tissue and fat distribution: comparison between normal men and women by magnetic resonance imaging. Thorax 1999;54:323-8.

22. Ucar FI, Uysal T. Orofacial airway dimensions in subjects with Class I malocclusion and different growth patterns. Angle Orthod 2011;81:460-8.

23. Dancey DR, Hanly PJ, Soong C, et al. Gender differences in sleep apnea: the role of neck circumference. Chest 2003;123:1544-50.

24. Tulgar S, Boga I, Cakiroglu B, et al. Short-lasting pediatric laparoscopic surgery: Are muscle relaxants necessary? Endotracheal intubation vs. laryngeal mask airway. J Pediatr Surg 2017;52:1705-10.

25. Bhardwaj N, Yaddanapudi S, Singh S, et al. Insertion of laryngeal mask airway does not increase the intraocular pressure in children with glaucoma. Paediatr Anaesth 2011;21:1036-40.

26. American Society of Anesthesiologists Task Force on Management of the Difficult Airway. Practice guidelines for management of the difficult airway: an updated report by the American Society of Anesthesiologists Task Force on Management of the Difficult Airway. Anesthesiology 2003;98:1269-77.

27. Rose DK, Cohen MM. The airway: problems and predictions in 18,500 patients. Can J Anaesth 1994;41:372-83.

28. Kaplan A, Crosby GJ, Bhattacharyya N. Airway protection and the laryngeal mask airway in sinus and nasal surgery. Laryngoscope 2004;114:652-5.

29. Tang MY, Tang IP, Wang CY. Optimal Size AMBU(R) Laryngeal Mask Airway Among Asian Adult Population. Med J Malaysia 2014;69:151-5.

Cite this article as: Ren Y, Cao C, Liang X, Ju Z, Zhang L, Cui X, Wang G. Validation of manufacturers' laryngeal mask airway size selection standard: a large retrospective study. Ann Transl Med 2021;9(3):196. doi: 10.21037/atm-20-4838
30. Tham LC. Children and size of laryngeal masks. Can J Anaesth 1994;41:354.

31. Huang YH, Cherng CH. Optimal size selection of the classic laryngeal mask airway by tongue width-based method in male adults. J Chin Med Assoc 2014;77:422-5.

32. Nott MR. Appropriate size of the laryngeal mask airway in adults. Br J Anaesth 1998;81:657.

33. Gu Y, McNamara JA Jr, Sigler LM, et al. Comparison of craniofacial characteristics of typical Chinese and Caucasian young adults. Eur J Orthod 2011;33:205-11.

34. Alves M Jr, Franzotti ES, Baratieri C, et al. Evaluation of pharyngeal airway space amongst different skeletal patterns. Int J Oral Maxillofac Surg 2012;41:814-9.

35. Wang T, Yang Z, Yang F, et al. A three dimensional study of upper airway in adult skeletal Class II patients with different vertical growth patterns. PLoS One 2014;9:e95544.

36. Tan SM, Sim YY, Koay CK. The ProSeal laryngeal mask airway size selection in male and female patients in an Asian population. Anaesth Intensive Care 2005;33:239-42.

37. Simon LV, Torp KD. Laryngeal Mask Airway. StatPearls. Treasure Island (FL): StatPearls Publishing; 2020.

38. Zhu Y, Shen W, Lin Y, et al. Cricoid-mental distancebased versus weight-based criteria for size selection of classic laryngeal mask airway in adults: a randomized controlled study. J Clin Monit Comput 2019;33:759-65.

39. Haliloglu M, Bilgen S, Uzture N, et al. Simple method for determining the size of the ProSeal laryngeal mask airway in children: a prospective observational study. Braz J Anesthesiol 2017;67:15-20.

40. Ramachandran SK, Mathis MR, Tremper KK, et al. Predictors and clinical outcomes from failed Laryngeal Mask Airway Unique: a study of 15,795 patients. Anesthesiology 2012;116:1217-26. 
Table S1 Equation about model-based weight probability scores for LMA sizes

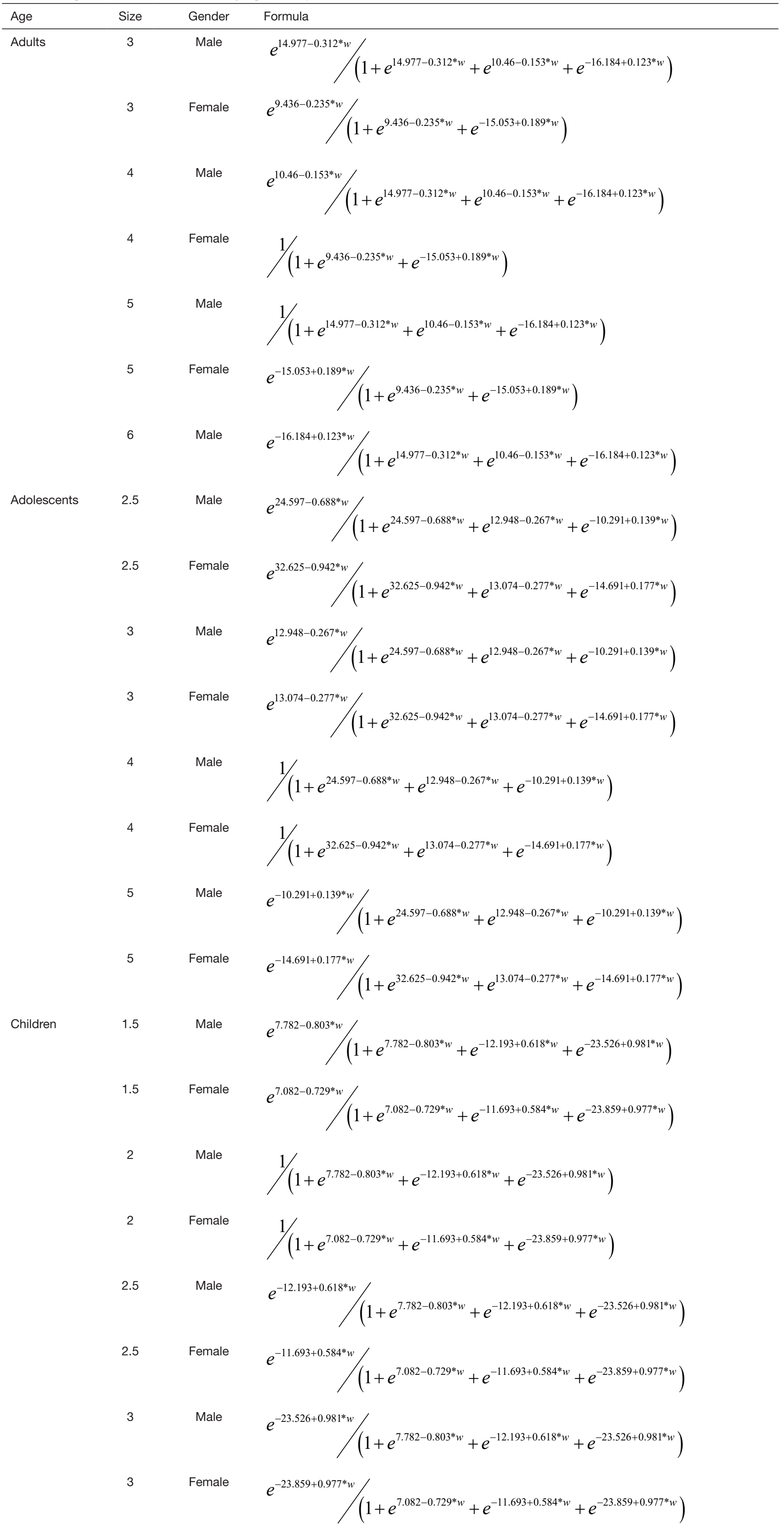

" $w$ " represents weight in the above table. We used "mlogit" in STATA software to calculate the coefficient for the multi-nominal logistic regression model. 